\title{
Theory and practice of laparoscopic surgery against omohyoid muscle syndrome
}

\author{
Zhipeng Sun ${ }^{\S}$, Yubing Zhu ${ }^{\S}$, Nengwei Zhang* \\ General Surgery Department, Peking University Ninth School of Clinical Medicine (Beijing Shijitan Hospital, Capital Medical \\ University), Beijing, China.
}

\begin{abstract}
Summary Omohyoid muscle syndrome (OMS) is a rare disease characterized as a protruding lateral neck mass feature during swallowing. Because there is a $5 \mathbf{c m}$ scar after traditionally surgery, we designed a laparoscopic surgery procedure to meet the cosmetic needs of patients. From the year 2006 to 2016, there were 3 patients diagnosed as omohyoid muscle syndrome that underwent laparoscopic surgery. Operative and postoperative follow-up data were summarized. Average surgery time was $35 \pm 13 \mathrm{~min}$. Average blood loss was $3 \pm 1 \mathrm{~mL}$. No case converted to open surgery. No major vessel or nerve damage complications occurred. After the surgery, the neck mass completely disappeared during swallowing, and there were no operative scars on the neck. All patients were discharged within 2 days. During the followup of a year, no recurrence occurred. In conclusion, the endoscopic procedure is suitable for OMS. It's a safe, effective and cosmetic surgery.
\end{abstract}

Keywords: Omohyoid muscle syndrome, laparoscopic surgery

\section{Introduction}

Omohyoid muscle syndrome (OMS) is presented by a mass without pain on the neck when the patient swallows and disappears after swallowing. The patient often feels discomfort and dysphasia when the mass appears. It's a rare disease. There are only few case-reports published about this disease. It's not able to be diagnosed by esophagus barium radiography or ultrasonography. The fine needle biopsy for the mass often results as muscle fibers atrophy, fibrosis or interstitial inflammation occurs. There is no typical pathology change. It's easily misdiagnosed.

In the normal condition (Figure 1A), the omohyoid muscle $(\mathrm{OM})$ consists of superior and inferior bellies united at an angle by an intermediate tendon (IT) and it passes behind the sternocleidomastoid (SCM) muscle. The inferior belly (InB) inclines forward and slightly

Released online in J-STAGE as advance publication August 25, 2017.

${ }^{\S}$ These authors contributed equally to this work.

*Address correspondence to:

Dr. Nengwei Zhang, 334 room, Administrative Building, Peking University Ninth School of Clinical Medicine (Beijing Shijitan Hospital, Capital Medical University). Tieyilu 10, Yangfangdian, Haidian District, Beijing 10038, China.

E-mail: zhangnw1@sohu.com upward and inserts into the IT. The superior belly ( $\mathrm{SuB}$ ) originates from the IT and inserts into the base of the hyoid bone $(\mathrm{H})$. The OM depresses the hyoid bone after it has been elevated during wallowing (1).

In OMS patients (Figure 1B), the most important pathology change is loosening of the IT tendon sheath (2). After that, the OM becomes shortened and fibrosis occurs because of atrophy of disuse and degeneration. When the patient swallows, the OM can't be extended, and the IT moves laterally and superiorly. The posterior clavicle margin of OM replaces IT as a new origin of force. When the patient swallows, the OM shortens like a string, and forms an X-shaped tent to elevate the SCM in the lateral neck during upward movement of the hyoid bone. The elevated SCM forms the mass in the neck.

This theory was acceptable for many scholars (35 ), but we could clearly see the thickened OM and the IT was still at its location contrarily when the patient swallowed. Obviously, the OM did not degenerate, but instead was more sturdy than the normal side. So the etiology responsible for OMS was not known for certain.

Transection of the omohyoid muscle is the standard treatment for this disease due to the pathophysiology change of OMS just described above (6). The prognosis is good except for a $5 \mathrm{~cm}$ scar on the neck. Because it's a benign disease, the only reason patients choose to undergo surgery is the cosmetic effect. So we designed 
this laparoscopic surgery procedure to meet the needs of patients.

\section{Materials and Methods}

\subsection{General information}

From the year 2006 to 2016, there were 3 patients diagnosed as omohyoid muscle syndrome and underwent laparoscopic surgery. Among them, 2 cases were male, 1 case was female. Age of the patients were from 26-40 years (35 average). Disease course was from 1 month to 2 years ( 5 month average). All of them didn't have any congenital cervical disease.

\subsection{Clinical presentation}

In the 3 cases, 2 presented left cervical mass, 1 presented right. Typical complain was a sense of mild

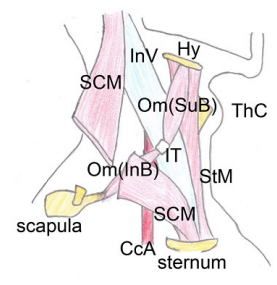

A

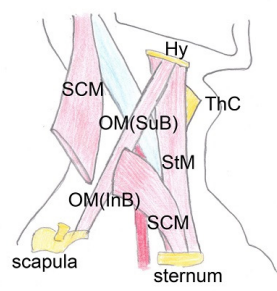

B
Figure 1. The anatomy and function of omohyoid muscle in normal and OMS patients. (A). In normal condition, omohyoid muscle (OM) consists of superior and inferior bellies that are united at an angle by an intermediate tendon (IT) and it passes behind the sternocleidomastoid (SCM) muscle. The inferior belly $(\mathrm{InB})$ inclines forward and slightly upward and is inserted into the IT. The superior belly ( $\mathrm{SuB}$ ) originates from the IT and is inserted into the base of the hyoid bone $(\mathrm{H})$. The OM depresses the hyoid bone after it has been elevated during swallowing. (B). In OMS patients, the most important pathology change is loosening of the IT tendon sheath. After that, the OM atrophies from disuse and degeneration happens. The OM becomes short and fibrotic. When the patient swallows, the OM can't be extended, the IT moves laterally and superiorly. The posterior clavicle margin of OM replaces IT as a new origin of force, When the patient swallows, the OM shortens like a string, forming an X-shaped tent to elevate the SCM in the lateral neck during upward movement of the hyoid bone. The elevated SCM forms the mass in the neck. dysphasia or a foreign body sensation in the throat. The history was usually several months. There was also an awareness of a mass appearing in the lower part of the neck when swallowing. Progression of symptoms was noticed. Voice was normal. There was also absence of distinct events that might have precipitated the onset of symptoms. A family history of similar symptoms was absent.

Physical examination showed no positive finding when the patient was not swallowing (Figure 2A). When the patient swallows (Figure 2B), a transient swelling arises up on the neck over the junction of the upper two thirds and the lower third of the sternomastoid muscle. The protruding of the mass coincides with the elevation of the throat, reaching its climax at the moment when the throat is uppermost. With the return of the hyoid, the OM shrinks to their original resting positions, and the mass also disappears. No further trace of the mass could then be discerned until the patient swallows again. Special maneuvers like the Valsalva maneuver or tongue protrusion are unrewarding.

\subsection{Assistant examination}

Blood, Urine tests, liver and kidney function were normal. Upper aerodigestive tract endoscopy, plain radiographs of the neck and thoracic inlet, and routine ultrasound, were normal. Computed tomography (CT) scan of the neck showed the inferior belly of the OM on the diseased side (Figure 3A) was obviously sturdier than that on the normal side (Figure 3B) by $1.15 \mathrm{~cm} v s$. $0.53 \mathrm{~cm}$ in diameter. The internal jugular vein of the diseased side was dilated compared with the normal side (Figure 3C) by $1.99 \mathrm{~cm} v s .0 .98 \mathrm{~cm}$ in diameter.

\subsection{Surgical procedure of laparoscopic surgery (Video 1)}

The patient was placed in a supine position with neck slightly extended under general anesthesia. A $10-\mathrm{mm}$ curved skin incision was made at the upper margin of mammary areolas. Diluted adrenalin solution (1:500) was injected into the subcutaneous space in the chest wall and in the subplatysmal space of the neck in order to establish the trocar space and prevent bleeding during
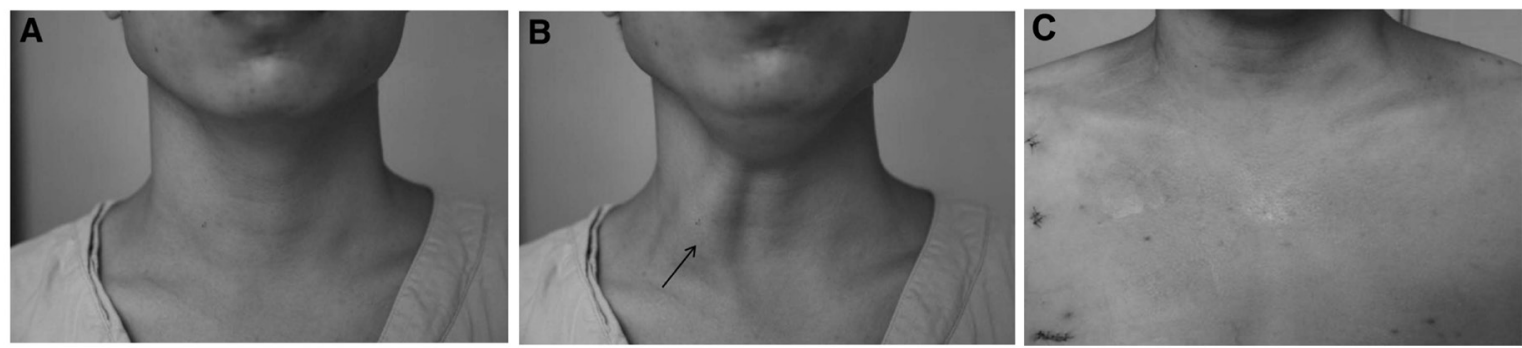

Figure 2. The Photograph of the patient resting, swallowing and after surgery. The features of the patient in the resting (A) and swallowing (B) are shown in the figure. The arrow points to the protruding mass. After surgery (C), the neck mass completely disappeared during swallowing, and there were no operative scars on the neck. 

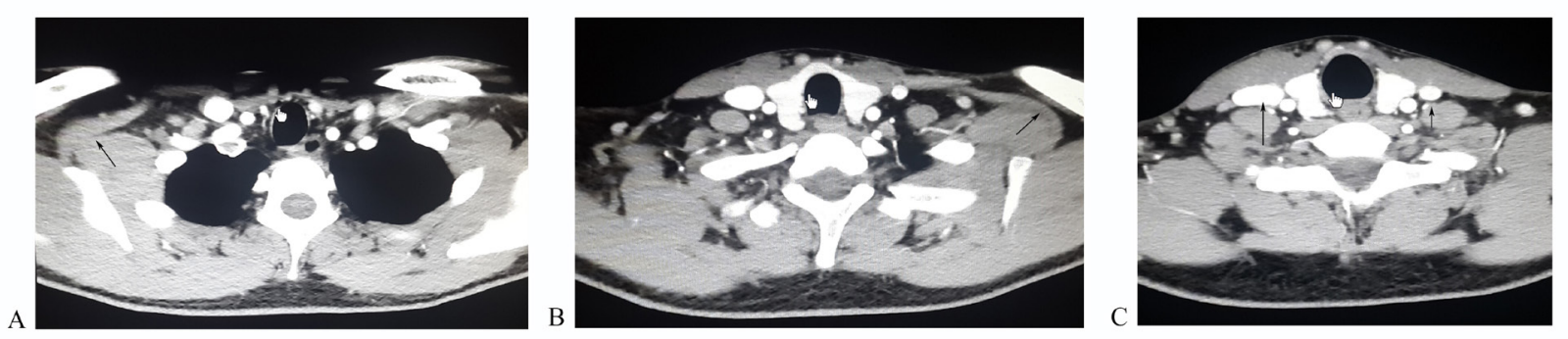

Figure 3. Computed tomography (CT) scan on the inferior belly of OM and internal jugular vein. Computed tomography (CT) scan of the neck showed the inferior belly of the OM on the diseased side (Figure 3A) was obviously sturdier than that on the normal side (Figure 3B) by $1.15 \mathrm{~cm} v s .0 .53 \mathrm{~cm}$ in diameter. The arrow points to the inferior belly of the OM. The internal jugular vein of the diseased side was dilated compared with the normal side (Figure 3C) by $1.99 \mathrm{~cm} v s .0 .98 \mathrm{~cm}$ in diameter. The arrow points to the internal jugular vein.

blunt dissection. The subcutaneous space was dissected bluntly using a dissector. Then a 10-mm trocar was inserted. Carbon dioxide was insufflated through the trocar at a pressure of 6-8 $\mathrm{mmHg}$, The endoscope was inserted into the subplatysmal space. Two additional trocars double $5 \mathrm{~mm}$ trocars below the axilla were inserted into the space. The remaining connective tissue in the space was dissected endoscopically using an electronic hook, and an adequate operative space above the sternocleidomastoid muscle was created. We isolated the sternocleidomastoid muscle. The color of the omohyoid muscle was pale and dark red, the muscle was obviously fibrotic and tightly adhered to the sternocleidomastoid muscle. The omohyoid muscle was dissected transversely at the upper and lower border of the sternocleidomastoid muscle by harmonic scalpel and electronic hook. Then the surgery was done.

Video 1 shows the surgical procedure of laparoscopic surgery against OMS (http://pan.baidu.com/s/ldELsA33). The patient was placed in a supine position. At the upper margin of mammary areolas, the endoscope was inserted. Two additional trocars double $5 \mathrm{~mm}$ trocars below the axilla were inserted into the subplatysmal space. Adequate operative space above the sternocleidomastoid muscle was created. The sternocleidomastoid muscle was isolated. The omohyoid muscle was dissected transversely at the upper and lower border of the sternocleidomastoid muscle by harmonic scalpel and electronic hook. Then the surgery was done.

\section{Results}

Average surgery time was $35 \pm 13 \mathrm{~min}$. Average blood loss was $3 \pm 1 \mathrm{~mL}$. and no case converted to open surgery. No major vessel or nerve damage complication occurred. There were no scars on the neck (Figure 2C). All the patients were discharged within 2 days. No recurrence occurred during the follow-up of a year.

\section{Discussion}

OMS, also called omohyoid sling syndrome, is a rare disease. The first report of a patient with OMS was in 1980 (7), a similar terminology, omohyoid syndrome, was first used in a report published in The Lancet in

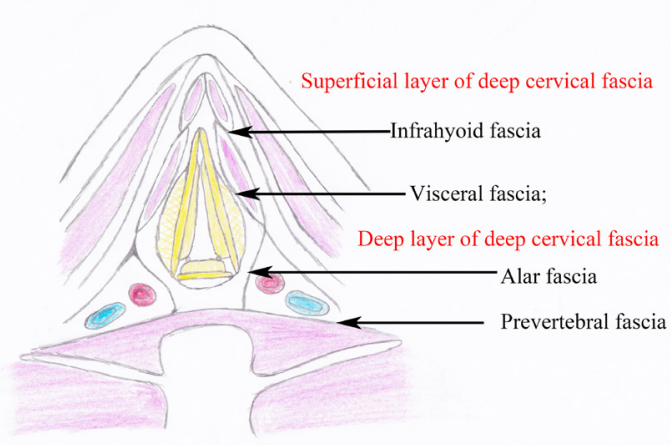

Figure 4. The diagraph of deep cervical fascia. The sarcolemma of OM consisted of a superficial layer of deep cervical fascia. At the same time this fascia also contributed to the anterior wall of the internal jugular vein sheath.

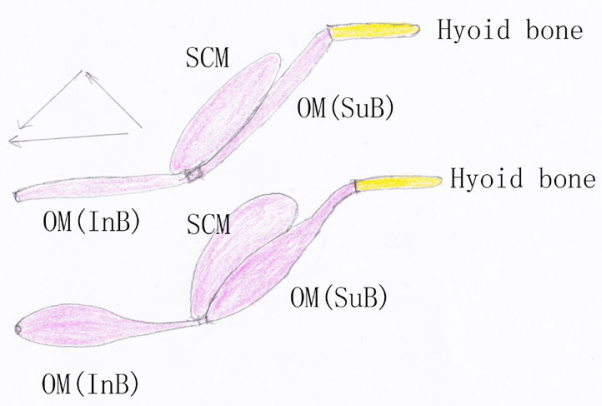

Figure 5. The diagraph of the etiology for OMS we thought. The etiology of OMS should be IT adhesion with the SCM, when swallowing, the OM should counteract part of the SCM forces. This resulted in OM compensatory sturdiness, and finally OM sturdy enough to elevate the SCM.

1969 to describe a case with characteristic symptoms, including pain and tenderness in the neck, voice changes, and swallowing difficulties most likely due to acute spasm or cramping of the omohyoid muscle. However, the patient did not show any mass in the neck during swallowing. Thus, this case is not compatible with the current concept of OMS (8).

From our clinical observation, we found patients who suffered from OMS didn't show OM degeneration and elevated IT as classic etiology. Instead, the OM on the diseased side was sturdier than the normal side. 
The sarcolemma of OM consisted of a superficial layer of deep cervical fascia. At the same time this fascia also contributed to the anterior wall of the internal jugular vein sheath (Figure 4). Partial function of the $\mathrm{OM}$ was dilating the internal jugular vein. So from the CT scan, we found that the internal jugular vein was obviously dilated compared with the normal side. This phenomenon also identified that the etiology of OMS was not OM degeneration as a classic hypothesis. From our observation, we thought the etiology should be IT adhesion with SCM, when swallowing, the OM should counteract part of the SCM forces (Figure 5). This resulted in $\mathrm{OM}$ compensatory sturdiness, and finally $\mathrm{OM}$ sturdy enough to elevate the SCM.

The key clinical finding of OMS is the appearance of a transient lower lateral neck mass during swallowing due to dysfunction of the omohyoid muscle. Physical examination characteristically showed no positive finding when the patient was not swallowing. Most patients with OMS were treated by surgical transection of omohyoid muscle. The procedure leaves $5 \mathrm{~cm}$ or longer scar on the neck. Botulinum toxin injection to omohyoid muscle under ultrasonography guidance for OMS could offer an effect of omohyoid muscle dilation without operative scars on the neck, but it was not reported whether OMS would recur or if another injection was required (9). Theoretically, the paralysis of the degenereted omohyoid muscle caused by botulinum toxin couldn't be complete and the effect couldn't be long lasting.

The use of endoscopic surgery on the neck is now widely used in thyroid and parathyroid glands (10). It's a safe and effective technique for benign disease with a good cosmetic effect (11). So we tried to use this technique in OMS patients. After surgery, the neck mass completely disappeared during swallowing, and there were no operative scars on the neck. The cosmetic effect was good.

In conclusion, we believe that the endoscopic procedure is suitable for this disease not only because of a safe and effective outcome but also a good cosmetic effect which is the reason why OMS patients underwent surgery.

\section{Acknowledgements}

The research was funded by Beijing Municipal Administration of Hospitals Clinical Medicine Development of Special Funding Support, code: XMLX201309. We thank the foundations and all the patients studied in this project.

\section{References}

1. Sukekawa R, Itoh I. Anatomical study of the human omohyoid muscle: Regarding intermediate morphologies between normal and anomalous morphologies of the superior belly. Anat Sci Int. 2006; 81:107-114.

2. Kim L, Kwon H, Pyun SB. Pseudodysphagia due to omohyoid muscle syndrome. Dysphagia. 2009; 24:357361.

3. Lee AD, Yu A, Young SB, Battaglia PJ, Ho CJ. Omohyoid muscle syndrome in a mixed martial arts athlete: A case report. Sports Health. 2015; 7:458-462.

4. Sun ZP, Zhu YB, Zhang NW. Laparoscopic omohyoid muscle transection surgery: A novel procedure against omohyoid muscle syndrome. Chin Med J (Engl). 2016; 129:604-605.

5. Zhu B, Li K, Li D, Amin B, Zhang D, Liu C. Endoscopic surgery for omohyoid muscle syndrome. J Laparoendosc Adv Surg Tech A. 2015; 25:672-674.

6. Wong DS, Li JH. The omohyoid sling syndrome. Am J Otolaryngol. 2000; 21:318-322.

7. Ye BY. Omohyoid muscle syndrome: Report of a case. Chin Med J (Engl). 1980; 93:65-68.

8. Zachary RB, Young A, Hammond JD. The omohyoid syndrome. Lancet. 1969; 2:104-105.

9. Su PH, Wang TG, Wang YC. Ultrasound-guided injection of botulinum toxin in a patient with omohyoid muscle syndrome: A case report. J Clin Ultrasound. 2013; 41:373376.

10. Kitano H, Fujimura M, Kinoshita T, Kataoka H, Hirano M, Kitajima K. Endoscopic thyroid resection using cutaneous elevation in lieu of insufflation. Surg Endosc. 2002; 16:8891.

11. Gagner M, Inabnet BW, 3rd, Biertho L. Endoscopic thyroidectomy for solitary nodules. Ann Chir. 2003; 128:696-701.

(Received July 14, 2017; Revised August 9, 2017; Accepted August 15, 2017) 